\title{
Gel-Based Hippocampal Proteomic Analysis 2 Weeks following Traumatic Brain Injury to Immature Rats Using Controlled Cortical Impact
}

\author{
Ashley R. Kochanek ${ }^{b, c}$ Anthony E. Kline ${ }^{c, d}$ Wei-Min Gao b, c, e \\ Mandeep Chadhab,c Yichen Lai ${ }^{b, c}$ Robert S.B. Clark ${ }^{b, c}$ C. Edward Dixon ${ }^{\text {a, c }}$ \\ Larry W. Jenkins ${ }^{a, c}$ \\ ${ }^{a}$ Neurological Surgery, ${ }^{b}$ Critical Care Medicine, ${ }^{C}$ Safar Center for Resuscitation Research, ${ }^{d}$ Physical Medicine and \\ Rehabilitation, University of Pittsburgh, Pittsburgh, Pa., and ${ }^{\mathrm{e}}$ Department of Environmental Toxicology, Institute of \\ Environmental and Human Health, Texas Tech University, Lubbock, Tex., USA
}

\section{Key Words}

Hippocampus $\cdot$ Phosphorylation $\cdot$ Pyruvate $\cdot$ Protein oxidation - Mitochondria $\cdot$ Rat brain $\cdot$ Pediatric traumatic brain injury

\begin{abstract}
Traumatic brain injury (TBI) to postnatal day 17 rats has been shown to produce acute changes in hippocampal global protein levels and spatial learning and memory deficits. The purpose of the present study was to analyze global hippocampal protein changes 2 weeks after a moderate ipsilateral controlled cortical impact in postnatal day 17 rats using 2dimensional difference gel electrophoresis and mass spectrometry. Paired sham and ipsilateral injured hippocampal lysates were independently labeled with different fluorescent cyanine dyes and coseparated within the same immobilized $\mathrm{pH}$ gradient strips and slab gel based on isoelectric point and molecular mass. Significant changes in key proteins involved in glial and neuronal stress, oxidative metabolism, calcium uptake and neurotransmitter function were found 2 weeks after injury, and their potential roles in hippocampal plasticity and cognitive dysfunction were discussed.

Copyright $\odot 2006$ S. Karger AG, Basel
\end{abstract}

\section{KARGER}

Fax +41613061234

E-Mail karger@karger.ch

www.karger.com
(C) 2006 S. Karger AG, Basel

Accessible online at: www.karger.com/dne

\section{Introduction}

Childhood trauma resulting in traumatic brain injury (TBI) due to accidents and abuse is the major cause of death and dysfunction in young children and is a significant public health problem $[1,2]$. After TBI, complex changes to the immature central nervous system (CNS) occur due to injury processes interacting with ongoing brain development. This is important in both the acute and the recovery/regenerative phases of injury. For example, recent rodent studies in pediatric TBI and previously in hypoxia/ischemia have demonstrated age-dependent vulnerabilities of the developing brain where successful therapeutic manipulations used in adult rats are harmful to normal development [3]. These data suggest that the developing brain may respond to injury and treatment differently than the adult brain.

Many of the changes in response to brain injury and treatment ultimately occur at the protein level by alterations in protein expression/degradation, protein-protein interaction and protein functional activity. The examination of pediatric TBI CNS protein function is more complex since the brain progresses through developmental stages using many of the same intracellular protein signaling pathways that are frequently altered by injury 
[4]. Since injury-induced protein changes are superimposed upon developmental protein changes, it has been proposed that global proteomic approaches may be even more important in the study of experimental pediatric TBI [4].

Prior study has demonstrated the utility of both conventional and functional large-format 2-dimensional gel proteomic approaches in examining acute $(24 \mathrm{~h})$ injury-related protein changes in the controlled cortical impact (CCI) model in postnatal day (PND) 17 rats [4]. Based upon synaptic and behavioral functional development, this rodent age is approximately equivalent to a 7-month- to 1-year-old child [4-6]. In this 'acute' proteomic study of moderate pediatric TBI, decreases in oxidative and endoplasmic reticulum stress proteins were unexpectedly seen, as well as decreases in protein kinase B activity important in survival, growth, glucose metabolism and synaptic plasticity [4]. Interestingly, this injury results in spatial memory impairment when tested 2 weeks after injury [7, unpubl. observations]. Thus, 14-day survival after CCI is an important survival time to evaluate functionally important protein changes in this model that may be related to cognitive dysfunction. Based on these data, the present study evaluated global protein changes after the same moderate CCI injury in PND 17 rats using 2-dimensional difference gel electrophoresis (DIGE) and mass spectrometry 2 weeks following injury. The objective of the present study was to expand the postinjury time course that has previously been examined in order to understand the more chronic response of global protein changes after experimental pediatric TBI.

\section{Methods}

\section{Model}

Twelve PND 17 male Sprague-Dawley (Harlan) rats (approximately $35 \mathrm{~g}$ ) were randomized into sham or injury groups $(\mathrm{n}=$ 6/group). Animals underwent surgery to produce a unilateral 7$\mathrm{mm}$ circular craniotomy midway between the bregma and lambda suture lines with the medial edge of the craniotomy $1 \mathrm{~mm}$ lateral from the coronal suture over the left cortex under isoflurane anesthesia. A focal TBI to the left cortex was produced by moderate CCI using a 6-mm-diameter pneumatic piston device at a velocity of $4 \mathrm{~m} / \mathrm{s}$ and a brain deflection depth of $2 \mathrm{~mm}$ resulting in cortical damage but sublethal overt hippocampal injury $[4,8]$. Immediately after CCI, the bone flap was replaced with dental cement, the incision sutured and anesthesia discontinued. Core temperature was maintained at normothermia throughout surgery, and rat pups recovered in a warmed cubicle for several hours before subsequently being transferred back to their dams. The Institutional Animal Care and Use Committee at the University of Pittsburgh in compliance with National Institutes of Health guidelines approved the experimental protocol.

Two weeks after injury, animals were decapitated under $4 \%$ isoflurane anesthesia, and the rostral half of the left dorsal hippocampus ipsilateral to the impact was rapidly dissected and frozen in liquid nitrogen. Both sham and injured ipsilateral hippocampi were briefly rinsed in cold isotonic saline before freezing to reduce brain contamination with blood proteins. Frozen samples were stored at $-80^{\circ} \mathrm{C}$ for subsequent 2-dimensional gel analysis.

Parallel studies of 2-week histopathology in rat pups were not evaluated, but previous studies of ipsilateral impacted hippocampus with survival times of $24-72 \mathrm{~h}$ after injury have revealed no overt hippocampal neuronal death at this injury level in our PND 17 CCI model $[4,8]$.

\section{Protein Separation}

Frozen hippocampi were processed for protein separation and analysis using the Ettan 2-dimensional fluorescence DIGE system at Windber Research Institute Amersham Proteomics Facility (Somerset, Pa., USA). Briefly, independent sham $(n=6)$ and injured $(n=6)$ hippocampi ipsilateral to injury impact were individually solubilized, and each group was labeled with a different cyanine fluorescent dye (Cy3 for sham, Cy5 for TBI), mixed in pairs, and coseparated by 2 -dimensional electrophoresis. The DIGE method separately labels each hippocampal sample and then mixes one sham with one injured sample (paired sample) so that each paired sample is run together on one gel resulting in six 2-dimensional gels containing one sham and one injured paired sample [9]. First-dimension separation of samples, based on isoelectric point, was performed by isoelectric focusing. Second-dimension separation based on molecular mass was performed by vertical large-format SDS-PAGE. Each gel yielded two separate images representing a sham or injured sample when illuminated by different wavelengths of light (fig. 1,2). Gel images were digitally converted to grayscale images and transferred to a UNIX workstation for subsequent analysis of spot densities (fig. 2). The gels were also stained with Sypro Ruby to visualize the total protein in each gel from both samples.

\section{Image Analysis}

Semiquantitative image analysis was performed using BioImage 2-dimensional analysis software (version 6.3, Genomic Solutions, Inc., Ann Arbor, Mich., USA). Spots were identified, manually edited and matched between image pairs by defining common anchor points and applying a constellation-matching algorithm. After normalization of images using the ratio of total intensity of all matched proteins in each gel, differences in intensity were calculated for each spot on every pair of images [4].

\section{Statistical Analysis}

A mean of approximately 300 protein spots $(302 \pm 54)$ per sample $(\mathrm{n}=12)$ was resolved in each large-format $(22 \mathrm{~cm})$ gel. Approximately $87 \%(263 \pm 525)$ of the 300 spots were matched between gels from each sample pair. First, protein identification using gel matching with known databases was performed. Then, proteins found in all six gel pairs with mean changes (17 proteins) greater than $25 \%$ had their identities confirmed using mass spectrometry as well as some other proteins of interest. Statistical analysis using StatView software consisted of a nonparametric 

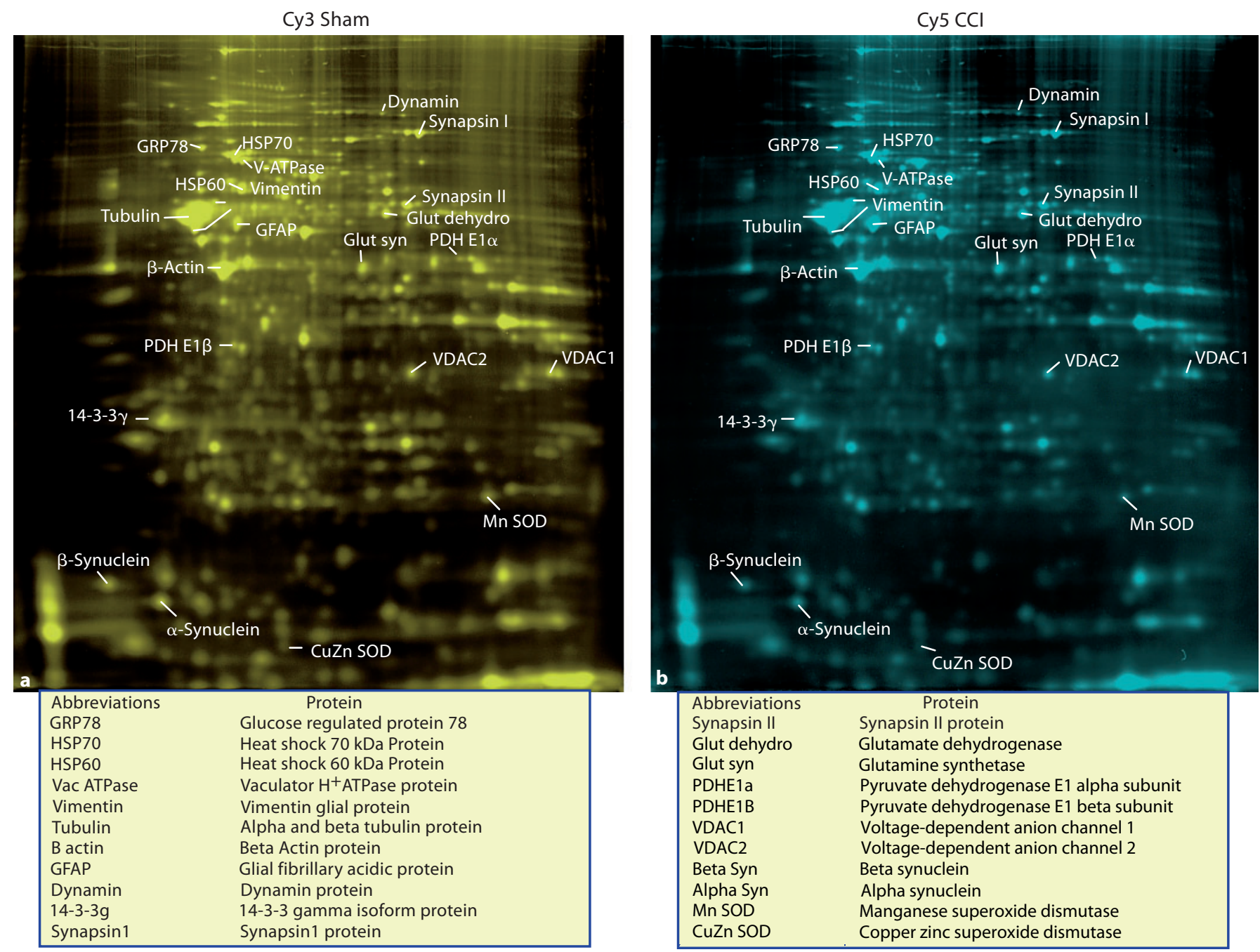

Fig. 1. Gels from a paired sample that have been colorized (colors are not the actual color of the Cy3 and Cy5 cyanine dyes) to make spot detection and annotation more visible. The relative positions and signal strength of the major proteins that showed a significant change 2 weeks after moderate CCI are shown in a paired sham (a) and CCI (b) sample for comparison.

one-sample sign test with a hypothesized value of 0 for all identified proteins with at least 1.25 -fold change across all six gel pairs. Results were reported as a list of spots that increased or decreased in intensity after injury at a given confidence level $(\mathrm{p}<0.05)$ (fig. 3).

\section{Protein Identification}

Protein spots were identified by gel matching with existing protein databases and mass spectrometry. Individual spots with greater than a $25 \%$ change in spot intensity or targeted proteins that changed in our acute study [4] across all six gel pairs were selected and punched from the original gels using Ettan Spot Picker automation, digested with trypsin and subjected to MALDI-TOF and/or Q-TOF mass spectrometry for identification confirmation at the Windber Research Institute Amersham Proteomic Facility. Protein sequence coverage and protein identification was determined using the Mascot search engine developed by Matrix Science (http://www.matrixscience.com/).

\section{Results}

\section{Protein Changes}

Proteins that showed significant changes are shown in a representative gel pair in figure 1, and the relative changes in spot intensity (mean \pm SEM) between groups 

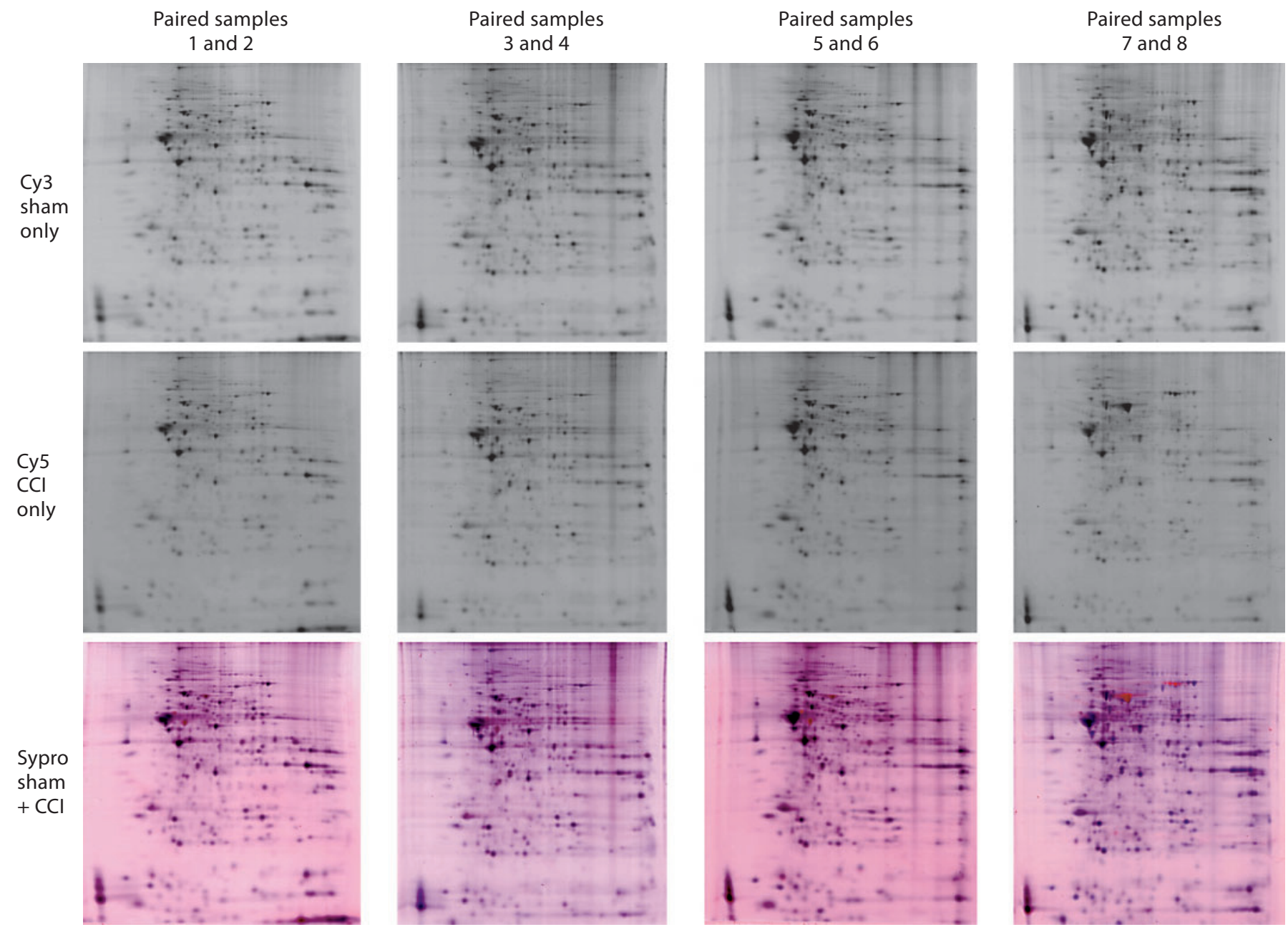

Fig. 2. Four gel pairs with individual grayscale images of the sham Cy3 dye and CCI Cy5 dye stained gels and a corresponding composite Sypro Ruby-stained gel of each paired sample. The Sypro Ruby-stained gel serves as a reference sample of both samples together to allow for the visualization of the spatial correlation between each paired sample and the image of the total proteins combined from each sample when imaged in the same gel. Gel quality used for the study and relative visual differences can be seen. Each column represents a single DIGE gel with a sham image (top row), injury image (middle row) and composite image (bottom row).

are shown in figure 3. Four paired gels from the six gel pairs used in the study are shown in figure 2 for relative visual comparisons of the representative raw gel data. The Sypro Ruby-stained gels serve as a reference sample of both paired sham and CCI samples together to allow for the visualization of the spatial correlation between each paired sample and the image of the total proteins combined from each sample when imaged in the same gel.

\section{Proteins Important in Energy Metabolism and Mitochondrial Function}

The most unexpected and potentially important protein changes found at this injury level were decreases in pyruvate dehydrogenase (PDH) E1 $\beta$ - and $\alpha$-subunits in CCI compared with sham (fig. 1, 3). PDH E1 $\beta$ was decreased by $-35 \pm 7 \%$ and PDH E1 $\alpha$ was diminished by $-31 \pm 6 \%$. In addition to PDH, two isoforms of the outer mitochondrial membrane porin protein family, decreases in voltage-dependent ion channel 1 (VDAC1) $(-17 \pm$ $4 \%)$ and VDAC2 $(-20 \pm 5 \%)$ were found (fig. 1, 3). 

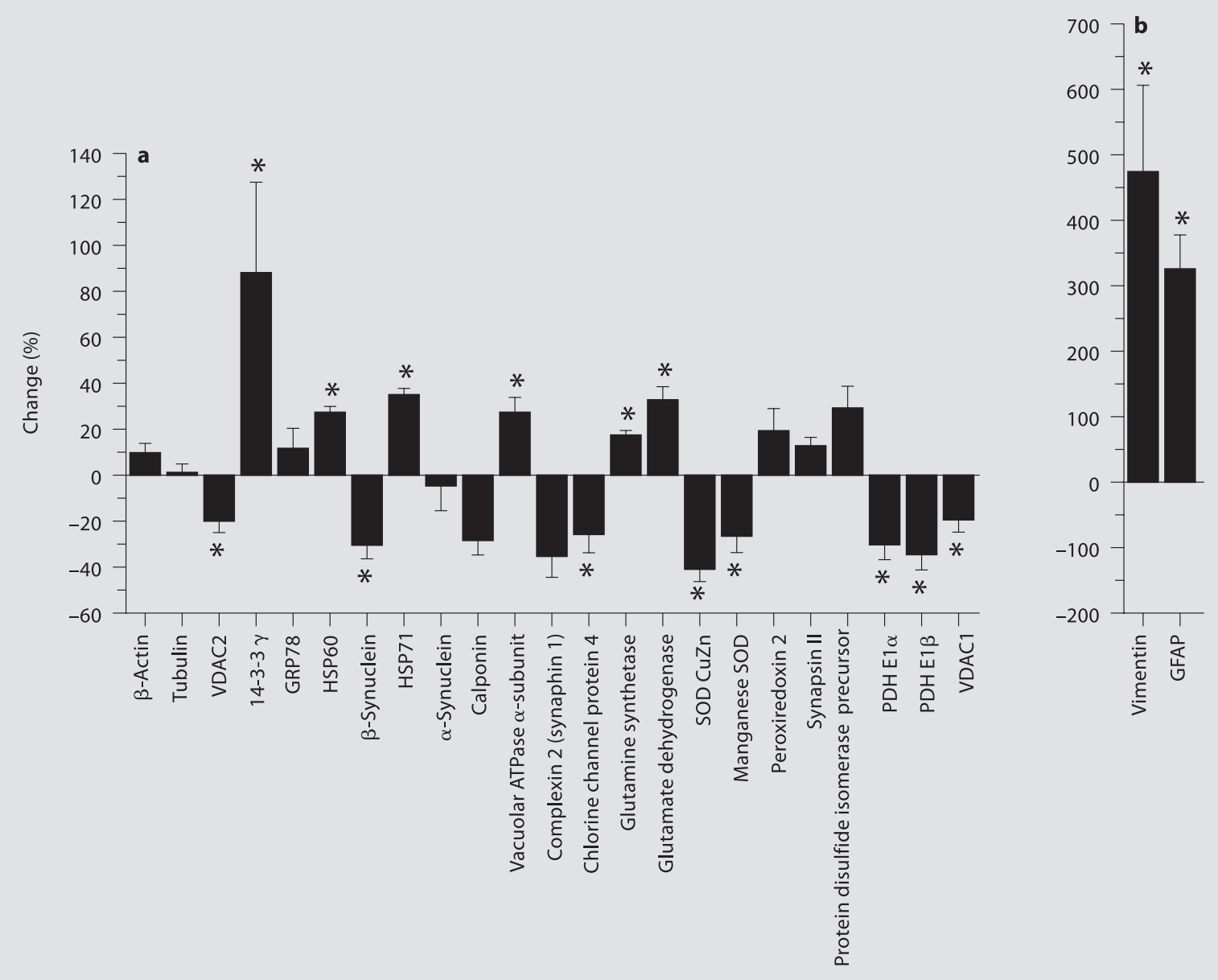

Fig. 3. a, b Group data are shown in the bar graphs and expressed as percentage of change from sham for relative differences between sham and CCI groups 2 weeks after TBI (mean \pm SEM). ${ }^{*} \mathrm{p}<0.05$.

\section{Synaptic Function Proteins}

No change was seen in the abundant synaptic proteins, synapsin I and II, or $\alpha$-synuclein. However, a significant increase in the important vacuolar ATPase $\alpha$-subunit (27 $\pm 7 \%$ ), critical in synaptic vesicle recycling and intracellular vesicle transport, was observed (fig. 1, 3). In contrast, a significant decrease in $\beta$-synuclein $(-31 \pm 6 \%)$ was seen (fig. 1, 3). A trend for increased synaphin 1 was found, but the protein was not resolved in all gel pairs and thus could not obtain statistical significance. The chloride intracellular channel protein 4 (CLIC4) which may also be involved in synaptic vesicle endocytosis (fig. 1, 3) was de- creased as well (-29 $\pm 7 \%)$. Two proteins involved in the glutamate-glutamine cycle were also resolved, namely, glutamate dehydrogenase and glutamine synthetase. Glutamate dehydrogenase $(33 \pm 5 \%)$ and glutamine synthetase $(17 \pm 2 \%)$ were moderately increased (fig. 1,3$)$.

\section{Oxidative Stress Proteins}

Changes in oxidative stress-related proteins were also found 2 weeks after injury. Decreases in both cytoplasmic CuZn superoxide dismutase 1 (SOD; $-42 \pm 5 \%$ ) and mitochondrial manganese SOD $2(-27 \pm 7 \%)$ were seen (fig. 1, 3). In contrast, nonsignificant changes for mito- 
chondrial peroxiredoxin 2 and endoplasmic reticulum protein disulfide isomerase were observed (fig. 1, 3).

\section{General Structural and Stress/Chaperone Proteins}

The ubiquitous cytoplasmic and mitochondrial stress proteins, the $70-\mathrm{kDa}$ heat shock protein (HSP70) and the mitochondrial $60-\mathrm{kDa}$ heat shock protein (HSP60), showed moderate increases of $35 \pm 3$ and $27 \pm 3 \%$, respectively (fig. 1,3). However, the endoplasmic reticulum stress protein, the glucose-regulated $78-\mathrm{kDa}$ protein (GRP78), did not show a significant change. The 14-3-3 $\gamma$-isoform displayed a moderate increase (88 $\pm 39 \%)$, while changes in actin and tubulin structural proteins were not observed. In contrast, the glial structural and stress proteins, vimentin and glial fibrillary acidic protein (GFAP), showed larger significant increases in spot density of $476 \pm 129$ and $323 \pm 52 \%$, respectively (fig. 1, 3 ), indicative of a glial stress response.

\section{Discussion}

Protein changes seen 2 weeks after pediatric brain injury with 2-D gels have to be cautiously viewed on the basis that they could be adaptive as well as maladaptive. Changes in 2-D gel protein levels could be regulated at a transcriptional, translational or posttranslational level and/or a consequence of altered mRNA processing and nucleo-cytoplasmic exchange. When more extended survival times are encountered, the likelihood of adaptive changes in protein function increases. Thus, it is sometimes impossible to differentiate adaptive from maladaptive protein changes without having a comprehensive understanding of the many other proteins involved in their function and regulation. Such data are seldom, if ever, provided by current proteomic approaches since each method only provides a partial and incomplete snapshot of the comprehensive global protein picture. As a consequence, caution should be exercised as to not overinterpret such protein data.

However, with this in mind, many of the protein changes observed in this study 2 weeks after moderate pediatric brain injury, while somewhat modest in magnitude, could be very important to the ongoing pathophysiology, recovery, and development of the injured immature brain. Significant changes in key proteins associated with the synaptic vesicle cycle, neurotransmitter synthesis, oxidative metabolism, stress response, antioxidant function and cellular signaling were found 2 weeks after brain injury. Of particular interest were the chronic

Hippocampal Proteomics 2 Weeks after Pediatric TBI changes seen in both glial and neuronal stress proteins, the PDH E1 $\alpha$ - and $\beta$-subunits and vacuolar ATPase and their potential roles in spatial memory dysfunction 2 weeks after injury. Interestingly, from the changes seen 2 weeks after moderate pediatric CCI, only $\mathrm{Cu} / \mathrm{Zn}$ SOD was also found to change at $24 \mathrm{~h}$ after moderate pediatric CCI [4]. These results document chronic global protein changes even 2 weeks after pediatric brain injury that may contribute to ongoing metabolic, signal transduction and behavioral morbidity. Additionally, even the limited fingerprint of protein changes seen with 2-D gel analysis after pediatric CCI suggests different patterns of protein changes compared with more acute time periods $(24 \mathrm{~h})$ analyzed previously with a comparable injury [4].

\section{PDH Changes}

$\mathrm{PDH}$ is the rate-controlling enzyme of cellular oxidative bioenergetics, and the PDH complex is composed of a multi-enzyme complex of many protein subunits [10]. Decreases were observed in the DIGE levels of both the $\mathrm{E} 1 \alpha$ - and $\beta$-subunits of PDH. Interestingly, PDH activity is modulated by phosphorylation (inactivation) and dephosphorylation (activation) of the PDH E1 $\alpha$-subunit $[10,11]$. Thus, decreases in the $\alpha$-subunit DIGE gel spot may be due to migration secondary to a changed phosphorylation status, which could either increase or decrease overall PDH activity 2 weeks after pediatric TBI in the present model. Currently, we have not yet examined the PDH E1 $\alpha$ phosphorylation status and do not know which possibility occurs. However, this does not account for the decrease in the PDH E1 $\beta$-subunit. The PDH complex is also quite sensitive to oxidation $[12,13]$, and, given our evidence for oxidative stress occurring at this time after pediatric injury (based upon changes in SOD), the mechanism for decreases in both PDH E1 $\alpha$ - and $\beta$-subunits may be the consequence of prior or continued protein oxidation.

If the observed PDH changes result in decreased PDH activity, this could potentially make the injured brain more sensitive to secondary insults that further challenge brain bioenergetic reserves. This finding was unexpected 2 weeks after injury and demonstrates that the window for such secondary complications may be persistent. In addition to the implications for changes in PDH activity for metabolic challenges to the injured brain, such changes may also affect PDH contributions to brain plasticity. The ability of mitochondria to regulate cellular-free calcium levels has been linked to mitochondrial PDH changes $[14,15]$, even under normal physiological conditions. 
In fact, a potential molecular mechanism for long-term potentiation (LTP) [16] was proposed due to changes in neuronal calcium buffering via altered mitochondrial PDH activity [14]. Decreased activity of PDH after pediatric TBI could also affect synaptic functions mediating LTP and long-term depression (LTD) by increasing the pool of monocarboxylates such as lactate and pyruvate, which are known to affect both hippocampal LTP and LTD [17]. Both LTP and LTD are believed by many to represent the electrophysiological correlates of molecular changes in synaptic efficacy used in CNS memory and learning formation [16].

Furthermore, a moderate decrease in basal function of $\mathrm{PDH}$ impacts downstream end product biosynthesis important for key neurotransmitter function. PDH-catalyzed reactions provide acetyl-CoA that can be converted to acetylcholine via the reaction with choline catalyzed by choline acetyltransferase or glutamate via the TCA cycle as well as transamination of $\alpha$-ketoglutarate, which is also the precursor for $\gamma$-aminobutyric acid [18]. Thus, changes in chronic PDH activity may alter the neurotransmitter pools of several key brain neurotransmitters 2 weeks after pediatric TBI, contributing to transmitter-related functional morbidity including learning and memory deficits.

\section{Glutamate-Glutamine Cycle}

Increases in glutamate dehydrogenase 1, also known as memory-related protein 2 as it is induced in the hippocampus during Morris water maze training (http:// ca.expasy.org/uniprot/P10860), and glutamine synthetase indicate changes in the glutamate-glutamine cycle after pediatric TBI. In contrast to neuronal pyruvate, astrocytic pyruvate can enter the TCA cycle as either acetyl-CoA or by carboxylation via pyruvate carboxylase. This generates a new molecule of oxaloacetate, which, when combined with acetyl-CoA, forms citrate and ultimately $\alpha$-ketoglutarate and glutamate in astrocytes [18]. Astrocytic glutamate is converted to glutamine, which is transported back to neurons via the glutamate-glutamine cycle and hydrolyzed to form neuronal glutamate. Glutamate released as a neurotransmitter is taken up by astrocytes where two thirds are converted to glutamine by glutamine synthetase and returned to neurons. One third of astrocytic glutamate is reconverted back to pyruvate via the TCA cycle [18]. Increases in glutamate dehydrogenase and glutamine synthetase following pediatric TBI 2 weeks after injury may represent an adaptation to handle the excessive neuronal glutamate release that occurred after injury. Additionally, based upon the well-docu- mented role of glutamate in LTP and learning and memory [16], changes in the glutamate-glutamine cycle may affect subsequent glutamate pool size and neurotransmission, and thus, contribute to altered cognitive function after pediatric TBI.

\section{Chronic VDAC Isoform Changes}

Three VDAC isoforms (VDAC1, VDAC2 and VDAC3) make up the porin protein family, which are the highest copy proteins of the mitochondrial outer membrane. Each isoform has slightly different channel functions which gate a number of ions and metabolites such as calcium and ATP [19]. VDAC1 has been proposed to play a role in the formation of the mitochondria permeability transition pore and cell death. VDAC1 changes have been implicated in a number of brain pathologies [20]. In contrast, the VDAC2 isoform has also been implicated in cell survival by binding to pro-death molecules such as BAK [21]. Thus, changes in porin levels may impact the resistance of mitochondrial mediated death cascades after pediatric brain injury. Since decreases in both VDAC isoforms were found, reduced VDAC1 may be pro-survival since decreases may diminish the formation of mitochondria permeability transition pore, and decreased VDAC2 may be pro-death since binding of pro-death molecules would be reduced. However, since hippocampal neuronal death was not a feature of the injury level used in the present study, other mitochondrial porin functions may be more important. Recent study has shown that spatial memory function is disrupted in porin-deficient mice [19], which may implicate a role of these more persistent changes in VDAC1 and VDAC2 in the cognitive deficits seen after pediatric TBI $[5,22$, 23].

\section{Synaptic Vesicle Cycle Proteins}

Vacuolar- $\mathrm{H}^{+}$ATPases (V-ATPases) are enzyme complexes that maintain the vesicle pool by reacidifying and refilling recycled vesicles with neurotransmitters. The hydrolysis of ATP by V-ATPase produces a proton gradient inside synaptic vesicles that is used by neurotransmitter-proton antiporters to uptake neurotransmitters [24]. $\mathrm{V}$-ATPases in the vesicle and presynaptic membrane may also regulate neurotransmitter release from mature vesicles by forming new vesicular fusions and ensuring that only fully filled vesicles are released. This preserves the quantal nature of neurotransmitter release [24]. Functional V-ATPases are also found in glia and contribute to transmitter uptake functions [25]. Changes in V-ATPases may further alter vesicle reacidification, refilling and 
recycling, resulting in changes in the resting vesicle pool. As a result, disruptions in fusion pore activity may cause abnormal release of unfilled vesicles, altered fusion events, altered neurotransmitter release and an altered response to neurological stimuli [24]. Increased V-ATPase levels following pediatric TBI may be a compensatory mechanism to reduce neurotransmitter dysfunction after TBI [26]. Any posttraumatic change in transmitter function likely influences synaptic plasticity and cognitive behavior.

In addition, decreases were observed in the CLIC4. While less is known about the functions and regulation of CLIC4, brain CLIC4 binds dynamin I and may also be involved in synaptic vesicle endocytosis, cell membrane remodeling and control of cell shape [27]. CLIC4 decreases after injury may reflect injury-related maladaptive synaptic vesicle endocytosis or membrane reorganization associated with learning and memory deficits observed after pediatric TBI.

\section{Stress Proteins}

Changes persisted at 2 weeks in the chaperone proteins HSP70 and HSP60. Following pediatric TBI, a rapid upregulation of HSP70 occurs [28]. HSP70 assists in a large variety of protein-folding activity in the cell, including folding of nascent proteins that could act in repair and regeneration and refolding of misfolded proteins damaged by injury. Evidence suggests HSP70 is neuroprotective [29]. HSP70 also interacts with HSP60 in the mitochondrial import of mitochondrial proteins [30]. HSP60, a mitochondrial matrix protein induced by stress, plays a key role in preserving the integrity of the mitochondria by properly folding proteins in the matrix and participating in protein import [30]. Increases in these proteins at 2 weeks could indicate a chronic, adaptive stress response persisting into chronic stages of injury. The lack of changes in glucose-regulated $78-\mathrm{kDa}$ protein indicates that endoplasmic reticulum stress is minimal compared with the cytosolic and mitochondrial compartments at this stage of injury.

\section{GFAP and Vimentin}

Large increases were observed in the expression of astrocyte intermediate filament proteins (GFAP and vimentin) 2 weeks after injury. These findings suggest a persistent glial stress response to pediatric TBI 2 weeks after injury. GFAP is a glial stress protein as well as a cytoskeletal protein [31]. Increases in GFAP and vimentin 2 weeks after injury may suggest sustained efforts by astrocytes to provide trophic support for recovery [32].

Hippocampal Proteomics 2 Weeks after

Pediatric TBI

\section{Synucleins}

Significant decreases were observed in $\beta$-synuclein while no change was observed in expression of the $\alpha$-isoforms after pediatric TBI. Interestingly, TBI has been implicated as a risk factor for neurodegenerative disease, and increases in the ratio of $\alpha$ - to $\beta$-synucleins, as observed in this model 2 weeks after injury, have been implicated as a potential mechanism for placing the brain at long-term risk of synucleopathies [33]. While the normal functions of synuclein proteins are still ill defined, preliminary evidence suggests that synucleins play an important role in the developing mammalian nervous system, particularly in synaptic function. These proteins are expressed in differentiated neuronal cells and are thought to support experience-dependent turnover of synaptic membranes, which may be important for enduring memory and learning functions [34]. Thus, changes seen in synuclein isoform levels and/or posttranslational modification status after pediatric TBI may affect synaptic and, in turn, cognitive function following injury.

\section{4-3-3 Activity}

There are seven 14-3-3 isoforms, including five major CNS isoforms, with at least one hundred 14-3-3-binding partners involved in signal transduction, cell cycle regulation, apoptosis, stress responses and malignant transformation [35]. 14-3-3 is a signaling protein involved in protein localization and regulation. Binding is primarily phosphorylation dependent; therefore, 14-3-3 interactions are largely regulated by the kinases and phosphatases that modulate the phosphorylation state of their target proteins [35]. To name but a few, protein kinase $\mathrm{A}$, protein kinase $\mathrm{C}$, calcium calmodulin kinase I, checkpoint kinase 1 , checkpoint kinase 2 , protein kinase $\mathrm{B}$ and ribosomal p90-kDa protein kinase phosphorylate sites mediate 14-3-3 binding; hence, the effect of 14-3-3 on a biological process will be related to the signaling pathway of the particular kinase family that it is interacting with at that time [35]. The increase in the 14-3-3 $\gamma$-isoform 2 weeks after pediatric TBI suggests a compensatory modulation of any of the above cell signaling pathways, many of which participate in cognitive processes [16]. In fact, 14-3-3 $\gamma$-isoforms have been implicated in cognitive signal transduction after TBI [36] and changes in 14-3-3 $\gamma$ could contribute to plastic and cognitive changes after pediatric TBI.

\section{SOD Activity}

Decreases were noted in both cytoplasmic and mitochondrial superoxide dismutases. Since CuZn SOD 1 (cy- 
toplasmic) and manganese SOD 2 (mitochondrial) are known antioxidant proteins, these changes have functional implications for the regulation of cellular concentrations of reactive oxygen species following TBI. The immature brain has been shown to be particularly susceptible to oxidative stress following an excitotoxic injury [37]. Decreases in superoxide dismutases may constitute a compromised capacity to reduce free radicals, and therefore, the brain may be more vulnerable to oxidative stress for weeks after pediatric TBI. Furthermore, since redox state regulates the activity of many of the signaling proteins involved in LTP and cognitive function $[16,38]$, any change in cellular redox status has important ramifications on cognitive signal transduction pathways $[16,38]$.
In summary, these findings collectively suggest longterm changes in a number of key proteins that may modulate behavioral and metabolic morbidity and/or participate in the repair phase of injury after pediatric TBI. Of particular interest are changes in PDH and synaptic vesicle proteins that have not been previously identified in TBI pathology.

\section{Acknowledgements}

The authors would like to thank Mr. Henry Alexander and Mr. Jeremy Henchir for their technical assistance. This work was supported by National Institutes of Health grant NS42648 (to L.W.J.). A T-32 grant (HD 40686) supported M.C. and Y.-C.L.

\section{References}

$\checkmark 1$ Adelson PD, Kochanek PM: Head injury in children. J Child Neurol 1998;13:2-15.

12 Prins ML, Hovda DA: Developing experimental models to address traumatic brain injury in children. J Neurotrauma 2003;20: 123-137.

3 Ikonomidou C, Stefovska V, Turski L: Neuronal death enhanced by N-methyl-D-aspartate antagonists. Proc Natl Acad Sci USA 2000;97:12885-12890.

4 Jenkins LW, Peters GW, Dixon CE, Zhang X, Clark RS, Skinner JC, Marion DW, Adelson PD, Kochanek PM: Conventional and functional proteomics using large format two-dimensional gel electrophoresis 24 hours after controlled cortical impact in postnatal day 17 rats. J Neurotrauma 2002;19:715-740.

5 Herschkowitz N: Neurological bases of behavioral development in infancy. Brain Dev 2000;22:411-416

6 Rice D, Barone S Jr: Critical periods of vulnerability for the developing nervous system: evidence from humans and animal models. Environ Health Perspect 2000; 108(suppl 3):511-533.

7 Stevenson KL, Skinner JC, Davis DS, Tran MP, Dixon CE, Kochanek PM, Jenkins LW, Adelson PD: Behavioral dysfunction in immature rats after controlled cortical impact (CCI). J Neurotrauma 2000;17:944.

$\checkmark 8$ Gao WM, Chadha MS, Kline AE, Clark RS, Kochanek PM, Dixon CE, Jenkins LW: Immunohistochemical analysis of histone $\mathrm{H} 3$ acetylation and methylation - evidence for altered epigenetic signaling following traumatic brain injury in immature rats. Brain Res 2006;1070:31-34.

9 Wu WW, Wang G, Baek SJ, Shen RF: Comparative study of three proteomic quantitative methods, DIGE, cICAT, and iTRAQ, using 2D gel- or LC-MALDI TOF/TOF. J Proteome Res 2006;5:651-658.
10 Patel MS, Korotchkina LG: Regulation of mammalian pyruvate dehydrogenase complex by phosphorylation: complexity of multiple phosphorylation sites and kinases. Exp Mol Med 2001;33:191-197.

11 Korotchkina LG, Patel MS: Probing the mechanism of inactivation of human pyruvate dehydrogenase by phosphorylation of three sites. J Biol Chem 2001;276:57315738.

12 Samikkannu T, Chen CH, Yih LH, Wang AS, Lin SY, Chen TC, Jan KY: Reactive oxygen species are involved in arsenic trioxide inhibition of pyruvate dehydrogenase activity. Chem Res Toxicol 2003;16:409-414.

13 Fiskum G, Rosenthal RE, Vereczki V, Martin E, Hoffman GE, Chinopoulos C, Kowaltowski A: Protection against ischemic brain injury by inhibition of mitochondrial oxidative stress. J Bioenerg Biomembr 2004;36: 347-352.

14 Browning M, Baudry M, Bennett WF, Lynch G: Phosphorylation-mediated changes in pyruvate dehydrogenase activity influence pyruvate-supported calcium accumulation by brain mitochondria. J Neurochem 1981; 36:1932-1940.

15 Baudry M, Kessler M, Smith EK, Lynch G: The regulation of pyruvate dehydrogenase activity in rat hippocampal slices: effect of dichloroacetate. Neurosci Lett 1982;31:4146.

16 Lynch MA: Long-term potentiation and memory. Physiol Rev 2004;84:87-136.

17 Yang B, Sakurai T, Takata T, Yokono K: Effects of lactate/pyruvate on synaptic plasticity in the hippocampal dentate gyrus. Neurosci Res 2003;46:333-337.
18 Hertz L: The astrocyte-neuron lactate shuttle: a challenge of a challenge. J Cereb Blood Flow Metab 2004;24:1241-1248.

19 Weeber EJ, Levy M, Sampson MJ, Anflous K, Armstrong DL, Brown SE, Sweatt JD, Craigen WJ: The role of mitochondrial porins and the permeability transition pore in learning and synaptic plasticity. J Biol Chem 2002;277:18891-18897.

20 Yoo BC, Fountoulakis M, Cairns N, Lubec G: Changes of voltage-dependent anion-selective channel proteins VDAC1 and VDAC2 brain levels in patients with Alzheimer's disease and Down syndrome. Electrophoresis 2001;22:172-179.

21 Cheng EH, Sheiko TV, Fisher JK, Craigen WJ, Korsmeyer SJ: VDAC2 inhibits BAK activation and mitochondrial apoptosis. Science 2003;301:513-517.

22 Adelson PD, Dixon CE, Kochanek PM: Longterm dysfunction following diffuse traumatic brain injury in the immature rat. J Neurotrauma 2000;17:273-282.

23 Prins ML, Povlishock JT, Phillips LL: The effects of combined fluid percussion traumatic brain injury and unilateral entorhinal deafferentation on the juvenile rat brain. Brain Res Dev Brain Res 2003;140:93-104.

24 Morel N: Neurotransmitter release: the dark side of the vacuolar- $\mathrm{H}^{+}$ATPase. Biol Cell 2003;95:453-457.

25 Philippe JM, Dubois JM, Rouzaire-Dubois B, Cartron PF, Vallette F, Morel N: Functional expression of V-ATPases in the plasma membrane of glial cells. Glia 2002;37:365-373.

26 Hayes RL, Jenkins LW, Lyeth BG: Neurotransmitter-mediated mechanisms of traumatic brain injury: acetylcholine and excitatory amino acids. J Neurotrauma 1992;9: S173-S187. 
27 Suginta W, Karoulias N, Aitken A, Ashley RH: Chloride intracellular channel protein CLIC4 (p64H1) binds directly to brain dynamin I in a complex containing actin, tubulin and 14-3-3 isoforms. Biochem J 2001;359: $55-64$.

-28 Lai Y, Kochanek PM, Adelson PD, Janesko K, Ruppel RA, Clark RS: Induction of the stress response after inflicted and non-inflicted traumatic brain injury in infants and children. J Neurotrauma 2004;21:229-237.

29 Lai Y, Du L, Dunsmore KE, Jenkins LW, Wong HR, Clark RS: Selectively increasing inducible heat shock protein 70 via TAT-protein transduction protects neurons from nitrosative stress and excitotoxicity. J Neurochem 2005;94:360-366.

30 Lister R, Hulett JM, Lithgow T, Whelan J: Protein import into mitochondria: origins and functions today (review). Mol Membr Biol 2005;22:87-100.
31 Lambert KG, Gerecke KM, Quadros PS, Doudera E, Jasnow AM, Kinsley CH: Activity-stress increases density of GFAP-immunoreactive astrocytes in the rat hippocampus. Stress 2000;3:275-284.

32 Dougherty KD, Dreyfus CF, Black IB: Brainderived neurotrophic factor in astrocytes, oligodendrocytes, and microglia/macrophages after spinal cord injury. Neurobiol Dis 2000;7:574-585.

-33 Uryu K, Giasson BI, Longhi L, Martinez D, Murray I, Conte V, Nakamura M, Saatman K, Talbot K, Horiguchi T, McIntosh T, Lee VMY, Trojanowski JQ: Age-dependent synuclein pathology following traumatic brain injury in mice. Exp Neurol 2003;184: 214-224.
4 Clayton DF, George JM: Synucleins in synaptic plasticity and neurodegenerative disorders. J Neurosci Res 1999;58:120-129.

35 Darling DL, Yingling J, Wynshaw-Boris A: Role of 14-3-3 proteins in eukaryotic signaling and development. Curr Top Dev Biol 2005;68:281-315.

36 Satchell MA, Zhang X, Kochanek PM, Dixon CE, Jenkins LW, Melick J, Szabo C, Clark RS: A dual role for poly-ADP-ribosylation in spatial memory acquisition after traumatic brain injury in mice involving NAD + depletion and ribosylation of 14-3-3gamma. J Neurochem 2003;85:697-708.

37 Peluffo H, Acarin L, Faiz M, Castellano B, Gonzalez B: $\mathrm{Cu} / \mathrm{Zn}$ superoxide dismutase expression in the postnatal rat brain following an excitotoxic injury. J Neuroinflammation $2005 ; 2: 1-12$.

38 Kamata H, Hirata H: Topical review: redox regulation of cell signalling. Cell Signal 1999;11:1-14. 